\title{
Author Index Vol. 11, 1994
}

Abdel-Wahab, E.-S. 16 Adler,G. 159,185 Aeberhard,P. 307 Aldea,G.S. 25 Alhan,E. 27 Alponat, A. 36 Al-Sharaf,K. 402 Amikura,K. 324 Ammann, R.W. 266 Andrén-Sandberg, Å. 402 Asprer, J. 20 Astudillo,E. 397 Axelson, J. 402

Baer,H.U. 355,371

Baize, M. 289

Banks, P.A. 219

Bassi,C. 451

Becker, Ch. 208

Beger,H.G. 359,377,414

Birkett,D. 25

Bloechle,C. 330

Bloom, E. 58

Bockman, D.E. 260

Bonenkamp, JJ. 86

Bornman,P.C. 341

Bramhall, S.R. 408

Broelsch, C.E. 330

Büchler, M.W. 137, 146, 208, 213, 244, 317, 355, 359, 371, 377, 390, 414, 445 Bunt, A.M.G. 86

Caldiron,E. 451 Calik,A. 27 Carr,T. 25 Carr-Locke, D.L. 365 Chattopadhyay, G. 20 Cinel,A. 27

Cremer,M. 289

Delhaye,M. 289

DeSantis,L. 451

Deutsch, A.A. 6

Deviere,J. 289

Di Carlo, V. 428

DiMagno,E.P. 299

Domínguez-Muñoz, J.E. 197

Dornschneider, G. 330

Duez,N. Ill

Dumonceau, J.-M. 289

Dutch Gastric Cancer Group 86

Ebert,M. 146 Engelhardt, R. 118 Erdogan, N. 36 Evrard,S. 10

Falconi, M. 451

Fernández-Cruz, L. 312,397

Finke,J. 118

Fornasiero, A. Ill

French Associations for Surgical Research

58 Frey,C.F. 324 Friess, H. 137, 146, 317, 359, 377,445 
Gansauge, F. 414 Glatting,G. 359 Gold, L.I. 149 Gonzalez, S. 397 Gotoh,M. 39 Gouzi,J.L. 58 Grant, E.A. 20 Grillenberger, K.G. 359 Gyr,K,E. 192,433

Halter, F. 133,345 Haugstvedt, T. 121 Heaton,N.D. 42 Hermans, J. 86 Hofbauer,B. 445

Houry, S. 68 Huguier, M. 68 Hürlimann, St. 438 Hurst, P.A. 42

Ihse,I. 402 Ikeda,M. 39 Imrie,C.W. 241 Inauen,W. 285 Ishizaka, T. 1 Isozaki,H. 78 Ito,T. 30

Iwase, K. 1 Izbicki,J.R. 330 Izukura, M. 30

Jaehne, J. 72

Kaibara,N. 93 Kalthoff,H. 156 Kamiike,W. 30 Kanai,T. 39 Katai,H. 99 Kawaguchi, T. 30

Keighley, M.R.B. 20 Khalil,A. 16 Kinoshita,T. 99 Kirchner,R. 104 Kirkham,N. 42 Kitajima, M. 64 Klöppel,G. 163 Knoefel, W.T. 330 Kobrin,M.S. 146 Korc,M. 137,146,149 Krenning, E.P. 456 Krige, J.E.J. 341 Kubota,T. 64 Kucuktulu,U. 27 Kumai, K. 64 Kupferschmid, J.P. 25 Kvols,L.K. 177,456 Kwekkeboom, D.J. 456

Labbé,F. 58 Landau, O. 6 Landen, S. 68 Lange, J. 256 Largiadèr, F. 303 Lawton,G.P. 274

Lemoine,N.R. 169 Lerch,M.M. 185 Link, K.-H. 414 Liptay,S. 159 Lise,M. Ill

Macintyre, I.M.C. 51 Maeta,M. 93 Malfertheiner, P. 197 Marchet,A. Ill Marescaux, J. 10

Maruyama, K. 99 Matsuda, H. 30 Meier, R.F. 433,461 Meyer, H.-J. 72 Miyata,M. 30

Modlin,LM. 274 Monden, M. 39 Mori,T. 39 Müller,M.W. 317 Mutter, D. 10

Naparkov, A. 99 Navarro, S. 312 Neoptolemos, J.P. 408 Nitti,D. Ill Nord,M. 10 Nudelman, I. 6

Obeid,T. 307 Odorico,J.S. 420 Ohata,T. 1 Ohgami, M. 64 Okajima,K. 78,99 Olfat,E.-S. 16

Ortiz, J. 20 Oshima, S. 1 Otani,Y. 64 Oya,M. 20

Papila,C. 36 Pauwels,S. 456 Pederzoli,P. 451 Pillasch,J. 414 Pour, P.M. 182 Prados,M. 312

Reber,H.A. 387 Reiss, R. 6 Reske,S.N. 359 Reubi,J.-C. 177,456 Rilinger, N. 414 Roeder,C. 156 Saenz, A. 312,397 Sahmoud,T. Ill

Sakon,M. 39 Salvador, L. 397 Sano,T. 99 Sarr,M.G. 251 Sartori,N. 451 Sasako,M. 86,99

Scheurer, U. 225 Schmassmann, A. 133, 345 Schmid, R.M. 159 Schmiegel, W. 156 Schmielau, J. 156 Schrag,H.-J. 213,244 Seiler, Ch.A. 390 Sgambati, S.A. 274 Shafik, A. 16 Shapira,O.M. 25 Sigurdsson, G.H. 230 Socci,C. 428 Sollinger, H.W. 420 Songun, I. 86 Stewart, A. 42 Stollfuss, J.C. 359 Stoupis, Ch. 208, 355

Takenaka, H. 1 Tarnawski, A. 133 Thumshirn, M. 192

Tominaga, H. 30 Triller,J. 350

Uhl,W. 208,213,244,377 Umeshita,K. 39 Uras,C. 36

Vandermeeren, A. 289 Velde, C.J.H. van de 86 Vock, P. 208, 355

Wagner, M. 371,390 Wakabayashi, G. 64 Watemberg, S. 6 Weidenbach, H. 159 Wheatley, A.M. 213 Wilker,D.K. 330 Wright, N. A. 142

Yagura, A. 1 Yamanishi, H. 30 Yoshidome,K. 30 Young, CJ. 420

Zimmermann, A. 337

466

Author Index 